\title{
Enfoque de la responsabilidad social empresarial en las pymes vinculadas al sector salud de la zona suroccidente de Bogotá"
}

Approach to corporate social responsibility in the small and medium companies of the sector health in the southwest zone of Bogota

Enfoque da responsabilidade social empresarial nas pyme vinculadas ao setor de saúde da zona sul oeste de Bogotá

Recibido: 4 de abril de 2017 Revisado: 31 de mayo de 2017 Aceptado: 16 de junio de 2017

Juany Amparo Camacho Kurmen***

Comité Distrital del Laboratorio de Bogotá

Vilma Esperanะa Camacho Kurmen****

Universidad Santo Tomás - Icontec

* Artículo de resultado de investigación. DOI: http://dx.doi.org/10.15332/s2145-1389.2017.0001.02

** Magíster (c) en Calidad y Gestión Integral. Especialista en Ciencia y Tecnología de Alimentos. Especialista en Gerencia de la Salud Pública. Bacterióloga y Laboratorista Clínica. Comité Distrital del Laboratorio de Bogotá, Universidad Santo Tomás. Correo electrónico: jamparocamacho@hotmail.com.

***Magíster (c) en Calidad y Gestión Integral. Especialista en Gerencia en Instituciones de Seguridad Social en Salud. Especialista en Epidemiologia. Licenciada en Química. Odontóloga. Universidad Santo Tomás. Correo electrónico: vecksanti@yahoo.es. 


\section{RESUMEN}

Este trabajo explora el enfoque de la responsabilidad social en las pymes vinculadas al sector salud de la zona suroccidente de Bogotá, como base para definir un instrumento que fortalezca la gestión de prácticas socialmente responsables. La metodología utilizada considera la aplicación de un cuestionario a 105 pymes, cuyos resultados fueron consolidados y analizados a través de una matriz en la que se contrastan enfoques teóricos de responsabilidad social a la luz de cuatro teorías: actuación social de la empresa, valor para el accionista, grupos implicados y ciudadanía empresarial y enfoques prácticos. Se consideraron las siguientes materias fundamentales de responsabilidad social: gobernanza, derechos humanos, prácticas laborales, medio ambiente, prácticas justas de operación, asuntos de consumidores y participación activa de la comunidad. Como resultados en lo referente a enfoques teóricos, la gestión socialmente responsable de las pymes encuestadas muestra una clara tendencia hacia el desarrollo de prácticas que se limitan al cumplimiento de lineamientos normativos. En lo que respecta a la evaluación de los aspectos prácticos se identificó un mayor nivel de desempeño en materia de participación activa y desarrollo de la comunidad, seguido por la adopción de elementos de gobernanza.

Palabras clave: responsabilidad social empresarial, pymes, sector salud.

\section{ABSTRACT}

This paper seeks to understand the approach to social responsibility in SMEs linked to the health sector of the southwestern area of Bogota, as a basis for defining a model that supports socially responsible management practices. The methodology considers the application of a survey of 105 SMEs linked to the health sector in the southwestern area of Bogota whose results were consolidated and analyzed by an array in which theoretical approaches to social responsibility are compared a Light four theories: social performance of the company, shareholder value, stakeholder groups and corporate citizenship and practical approaches: core subjects of social responsibility: governance, human rights, labor practices, environment, fair operating practices, issues of consumers and active community participation. According to the results, in terms of theoretical approaches, the socially responsible management of the surveyed SMEs shows a clear trend towards the development of practices that are confined to compliance with regulatory guidelines. With regard to the evaluation of the practical aspects a higher level of performance identified in active participation and the development of the community followed by the adoption of elements of governance.

Keywords: Corporate social responsibility, SMEs, health sector.

\section{RESUMO}

Este trabalho busca conhecer a abordagem da responsabilidade social nas PYME associadas ao setor da saúde da zona sudoeste de Bogotá, como base para definir uma ferramenta para fortalecer a gestão de práticas socialmente responsáveis. A metodologia inclui a aplicação de um questionário em 105 PYME, cujos resultados foram consolidados e analisados através de uma matriz por meio da qual se comparam enfoques teóricos da responsabilidade social no quadro de quatro teorias: atuação social da empresa, valor para 0 acionista, grupos implicados e cidadania empresarial e abordagens práticas: matérias fundamentais de responsabilidade social: governança, direitos humanos, práticas laborais, meio ambientes, práticas justas de 
operação, assuntos ligados aos consumidores e participação ativa da comunidade. Em relação sobre os enfoques teóricos, a gestão socialmente responsável das pyme interrogadas revela uma tendência para o desenvolvimento de práticas as quais se limitam a assegurar a conformidade com os requisitos reguladores.

No que se refere à avaliação dos aspectos práticos identificou-se um nível mais elevado de desempenho em matéria de participação e desenvolvimento da comunidade, seguida pela adoção de elementos de governança.

Palavras-chave: responsabilidade social corporativa, ferramenta de gestão, pyme, sector da saúde.

\section{INTRODUCCIÓN}

Las pequeñas y medianas empresas desempeñan un papel fundamental en la economía, especialmente por su contribución a la generación de empleo y al desarrollo de la comunidad donde se ubican. Este es un fenómeno que se observa a nivel mundial, tal como lo evidencian las estadísticas de la composición empresarial y de la generación de empleo (Baron, 2000). Según cifras del Ministerio de Comercio, Industria y Turismo; en Colombia a junio de 2014 las pymes representan el 3,5\% del tejido empresarial del país, además, las microempresas son el 96,4\% de las entidades, mientras que las grandes empresas alcanzan el $0,1 \%$.

Las microempresas generan un poco más del $50 \%$ del empleo y las pymes alcanzan el 30,5\% de la ocupación nacional. Según ACOPI, en el año 2003 de estas microempresas 170.000 son pymes del sector salud, esto significa que lo que pase con ellas es determinante para los procesos económicos y sociales a nivel nacional e internacional, de tal forma que la implementación de las prácticas de responsabilidad social en las pymes ayudará a su sostenibilidad en el tiempo (Saldaña, 2014).

Con este trabajo se cumple con los objetivos de la normatividad vigente regida por la Constitución Nacional de Colombia de 1991, el Ministerio de Protección Social, el Ministerio de Trabajo y el Ministerio de Medio Ambiente, Vivienda y Desarrollo Territorial, quienes en sus políticas de gestión recomiendan la aplicación de las prácticas de RSE. En especial se destaca esta implementación en el sector salud por la importancia de esta como elemento fundamental en el desarrollo del capital humano, cuyo eje central es el usuario y no los intereses económicos de las partes interesadas.

La inclusión de estas prácticas de RSE generará retos para las pymes estudiadas porque implica un cambio organizacional, la capacitación del personal, la contratación de consultores, la reforma de software y el fortalecimiento del trabajo con stakeholders. En esto radica la importancia del presente trabajo, pues a través de su desarrollo se propuso una herramienta de gestión fundamentada en las líneas de actuación social, con una estructura basada en el ciclo PHVA.

Esta herramienta apoyará el enfoque de gestión de las pymes para un desarrollo social como elemento diferenciador y fundamental para encaminar acciones que busquen trascender la prestación del servicio de salud, involucrando a los grupos de interés.

\section{METODOLOGÍA}

Los momentos en que se desarrolló la investigación fueron:

La planificación partiendo del problema de investigación: "Cuál es el enfoque de las acciones de 
responsabilidad social en las pymes del sector salud en la zona suroccidente de Bogotá, y una herramienta de gestión para fortalecer las prácticas de responsabilidad social para las pymes estudiadas". Se realizó una revisión bibliográfica de contextualización en términos de responsabilidad social, enfoques teóricos fundamentados en las teorías de responsabilidad social de Domenec Melé y el enfoque práctico definido por las materias fundamentales descritas en la norma ISO 26000:2010. Se seleccionó como instrumento la encuesta desarrollada por Giraldo (2011) y validada por Trilleros (2015). Se hizo un muestreo aleatorio simple y se aplicó a 105 pymes del sector salud de la zona suroccidente de Bogotá.

El diseño de la propuesta de una herramienta de gestión planteado se fundamentó en la reorientación de la articulación con las partes interesadas o stakeholders y en líneas de acción propuestas. La estructura se fundamenta en el modelo de gestión de mejora continua ciclo PHVA (ver figura 7).

Consolidación de la herramienta de gestión propuesta que genera valor para las pymes del sector salud como organizaciones con retos de sostenibilidad, mejora continua y competitividad (Mora, Miranda, Espinosa y Guarín, 2010).

\section{RESULTADOS Y DISCUSIÓN}

\section{Perfil empresarial}

A continuación, se presenta la descripción de las pymes según los servicios que presta, el tamaño de la empresa y la existencia de planeación estratégica.

Figura 1. Mapa de la distribución por zonas del Distrito de Bogotá

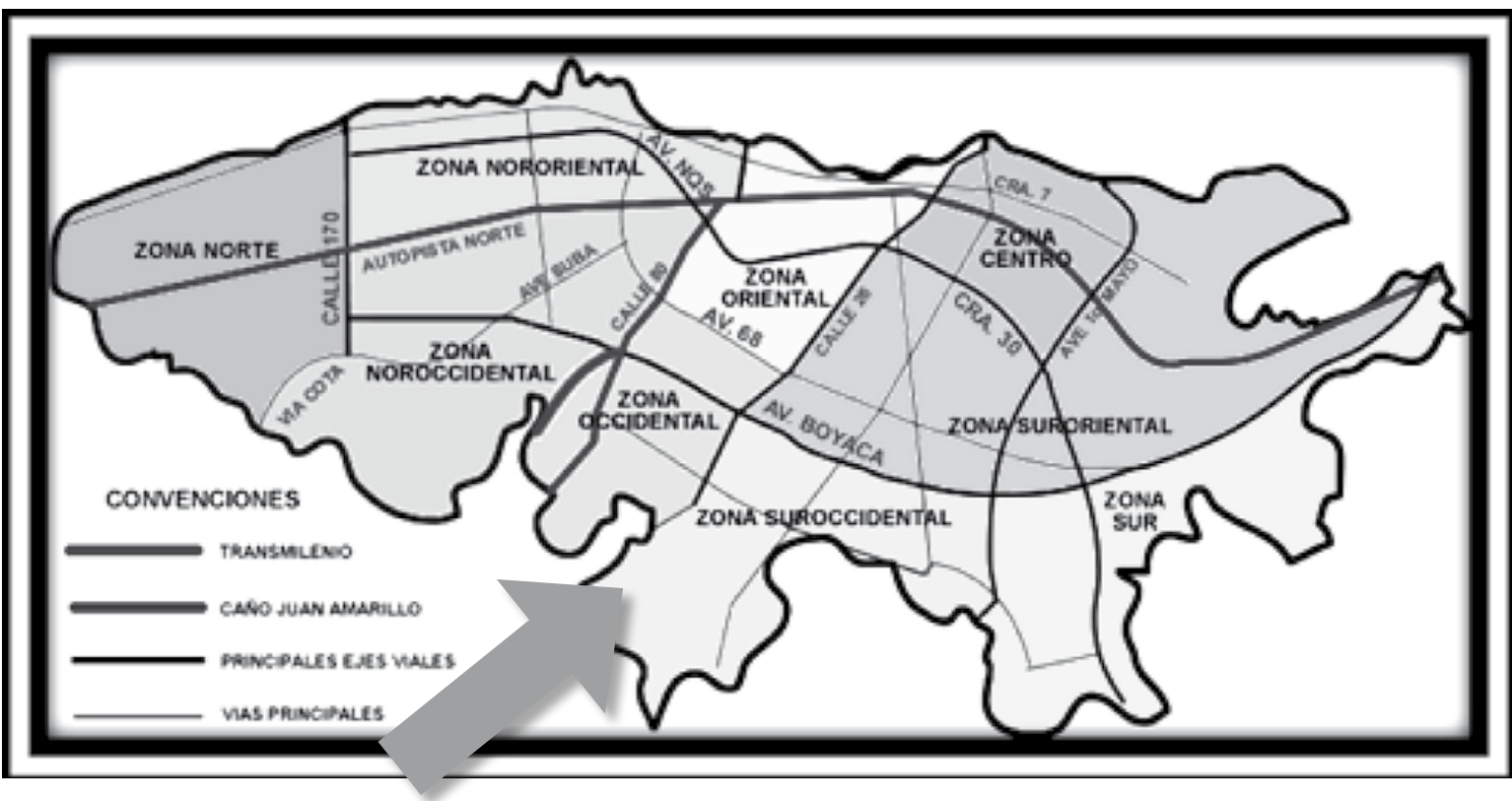

Fuente: mapas de distribución zonal del Distrito Capital 
Las pymes vinculadas al sector salud encuestadas fueron ubicadas en la zona suroccidente de Bogotá (figura 1), zona conformada por las localidades de Fontibón, Bosa y Kennedy. En esta zona se encuentran 144 pymes del sector salud habilitadas por la Secretaria de Salud de Bogotá.

De las 105 pymes encuestadas según el tipo de servicio que prestan, corresponden en un $20 \%$ a consultorios odontológicos, el $15 \%$ son laboratorios clínicos, el $18 \%$ prestan servicio de oftalmología y optometría, el $8 \%$ son consultorios médicos y el $39 \%$ prestan más de dos servicios (medicina, odontología, laboratorio, rayos $\mathrm{X}$, salud ocupacional, etc.).

Figura 2. Servicios que prestan las pymes encuestadas

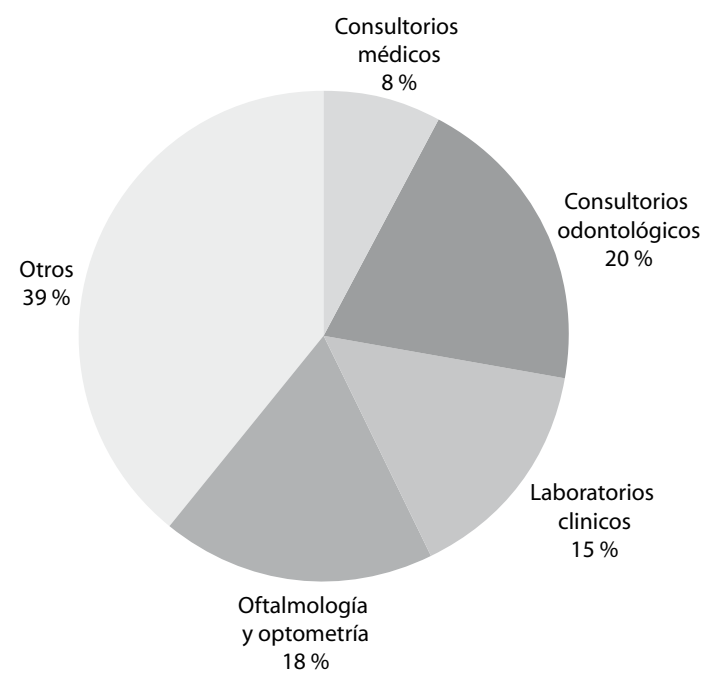

Fuente: elaboración propia (2015)

Las pymes estudiadas según el número de trabajadores son microempresas ( $<10$ trabajadores) en un $77 \%$, el 9 $\%$ son pequeñas empresas (entre 1 y 50 trabajadores) y el $14 \%$ son medianas empresas (entre 51 y 200 trabajadores) (Ley 905 de 2004) (figura 3). El ejercicio de la ética corporativa no solo es posible, sino necesario para todas las pymes independiente de su tamaño para que alcancen una posición y permanezcan con éxito en la economía global (Enderle, 2003).
Figura 3. Tamaño de las pymes

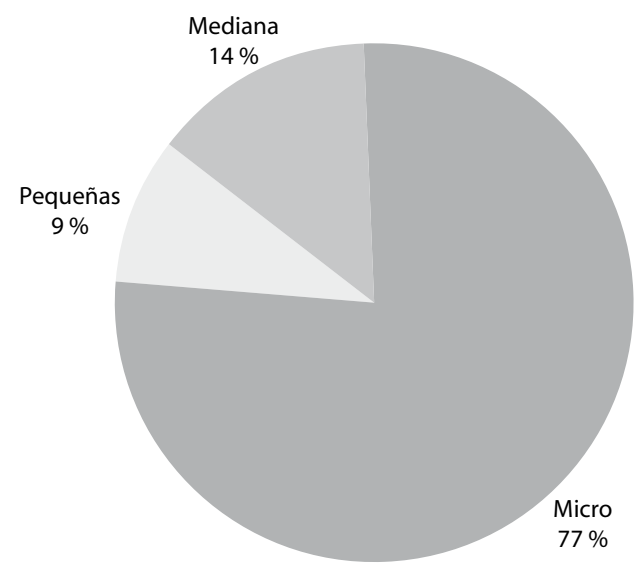

Fuente: elaboración propia (2015)

La identificación del enfoque de las acciones en responsabilidad social en las pymes estudiadas permite conocer el compromiso que afianza la conducta socialmente responsable del sector salud, y con ello propender en la mejora de su calidad y su sostenibilidad, razón por la cual se pregunta si estas pymes cuentan con una plataforma estratégica (figura 4), ya que desde esta se orientan las prácticas de responsabilidad social en la organización como uno de los pilares estratégicos que se aplican a través de una herramienta de gestión (Restrepo, 2009).

El $50 \%$ de las pymes respondió que sí cuentan con una plataforma estratégica, el $40 \%$ de las pymes encuestadas no contestó y el $10 \%$ respondió que no contaban con dicha plataforma. Estos resultados nos indican que la mayoría de las pymes encuestadas están motivadas y se les facilita implementar la herramienta de gestión propuesta en el desarrollo de esta investigación. El estudio ETHOS destaca la importancia de las herramientas de gestión de prácticas socialmente responsables porque promueven procesos de autorregulación y formación ética en las organizaciones (Restrepo, 2009). 
Figura 4. Plataforma estratégica las pymes

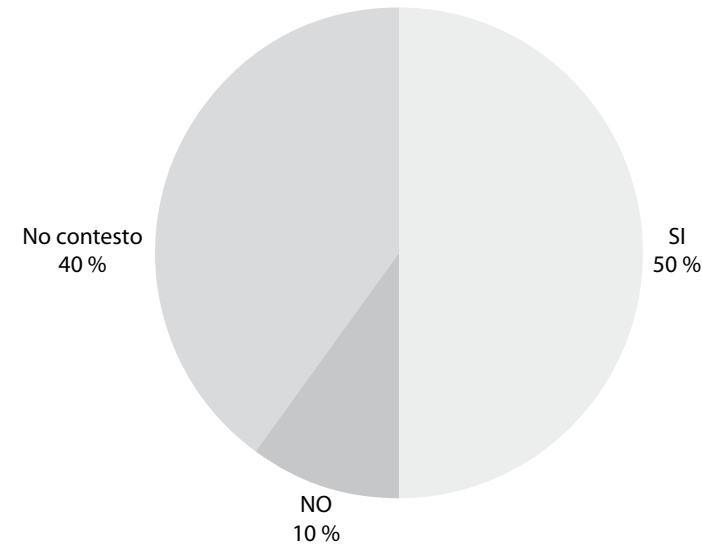

Fuente: elaboración propia (2015)

\section{Enfoques teóricos de las acciones de RSE en las pymes}

De acuerdo con los resultados obtenidos de la aplicación, de las 105 encuestas en las pymes del sector salud de la zona suroccidente de Bogotá, el $42 \%$ de las pymes desarrollan prácticas en responsabilidad social con un enfoque de valor para el accionista, mostrando una clara tendencia hacia el desarrollo de prácticas que se limitan al cumplimiento de lineamientos normativos. Seguido por un $25 \%$ por el enfoque de ciudadanía empresarial corporativa y en menor proporción se aprecia un $9 \%$ para el enfoque de actuación social de la empresa.

Figura 5. Enfoque teórico predominante de las pymes encuestadas

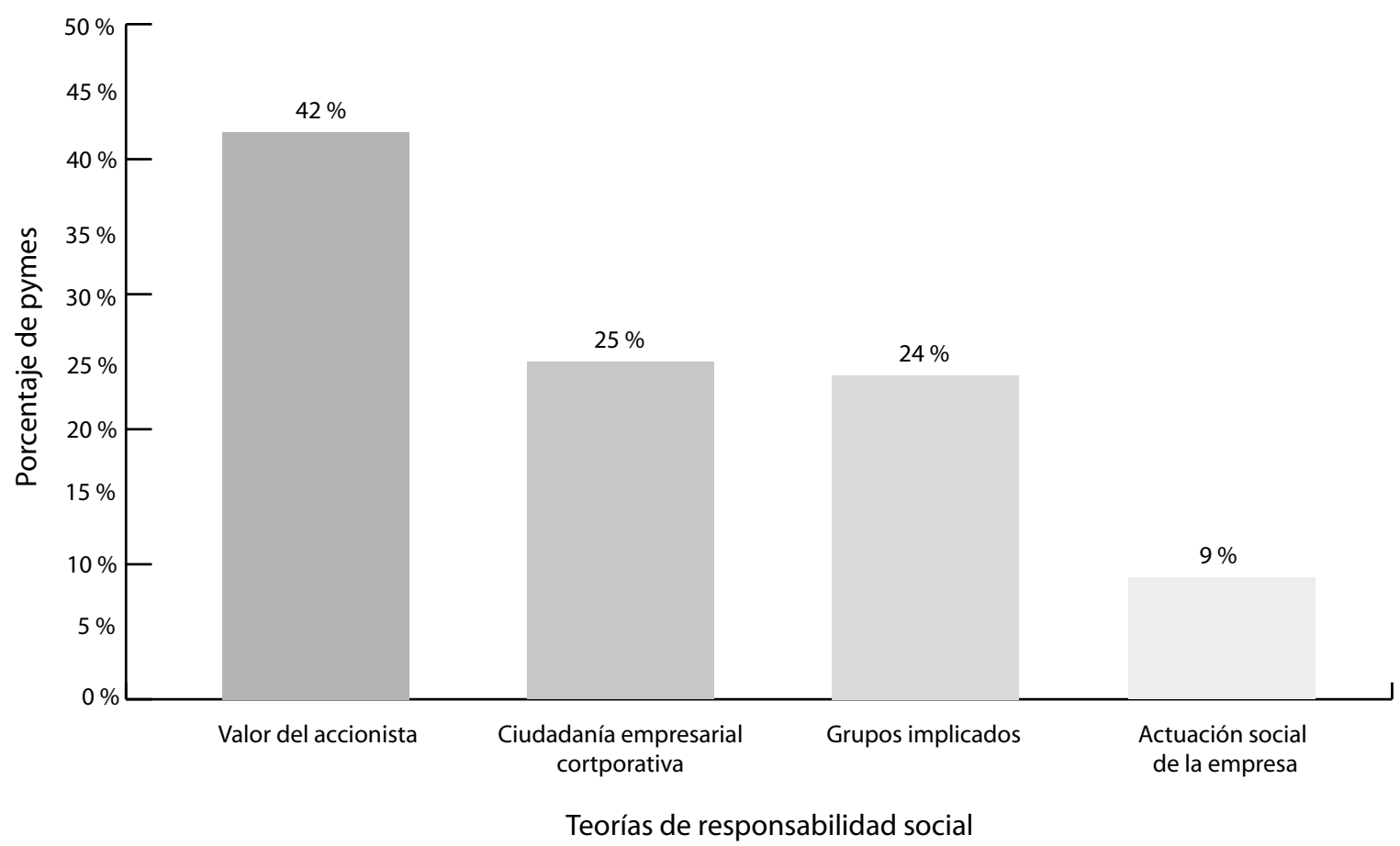

Fuente: consolidado de las encuestas aplicadas a pymes vinculadas al sector salud, zona suroccidente Bogotá (2015) 


\section{Enfoques prácticos de las acciones de RSE en las pymes}

En lo que respecta a la evaluación de los aspectos prácticos de la responsabilidad social (figura 6). En general se muestra que las pymes encuestadas presentaron un enfoque práctico de las materias fundamentales, entre un $19 \%$ y un $29 \%$, lo que indica que la herramienta de gestión propuesta fortalecerá y motivará el desarrollo de la gestión social en la comunidad donde desarrollan sus actividades.

Figura 6. Predominancia de los elementos prácticos adoptados por las pymes encuestadas

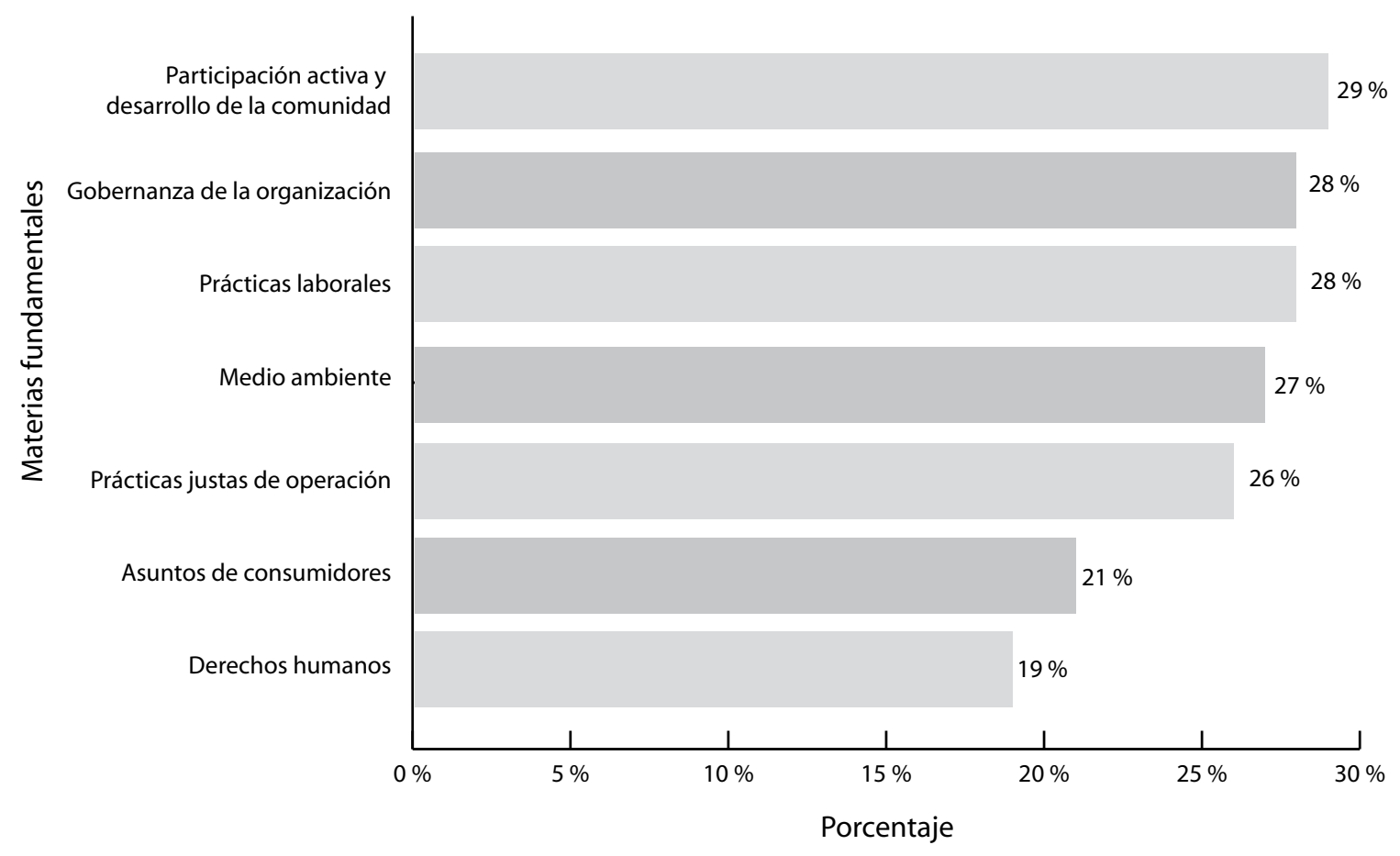

Fuente: consolidado de las encuestas aplicadas a pymes vinculadas al sector salud, zona suroccidente Bogotá (2015)

Se observa que solo un $29 \%$ de las pymes tienen un desempeño en materia de participación activa y desarrollo de la comunidad (Villegas y Quintanilla, 2012); de acuerdo con lo contemplado por la norma ISO 26000:2010, la participación activa y desarrollo de la comunidad es una invitación a las organizaciones a participar en la resolución de problemas de la comunidad de su entorno, a maximizar la creación de empleos, a promover la cultura y la educación, facilitar el acceso a la tecnología, la salud, la generación de riqueza de ingresos e inversión social.

\section{Herramienta de gestión de responsabilidad social para pymes vinculadas al sector salud}

La herramienta de gestión propuesta en esta investigación con base en los resultados de enfoque prácticos y teóricos, contiene una introducción, un campo de aplicación, una formulación por medio del ciclo de gestión o PHVA, una justificación, marco normativo y legal y las líneas de acción. 


\section{Campo de aplicación}

Dicha herramienta de gestión está orientada a proporcionar directrices a las pymes vinculadas al sector salud para planear, implementar, aplicar y mantener a través de la mejora continua un enfoque de gestión socialmente responsable.

Figura 7. Herramienta de gestión RSE para pymes sector salud

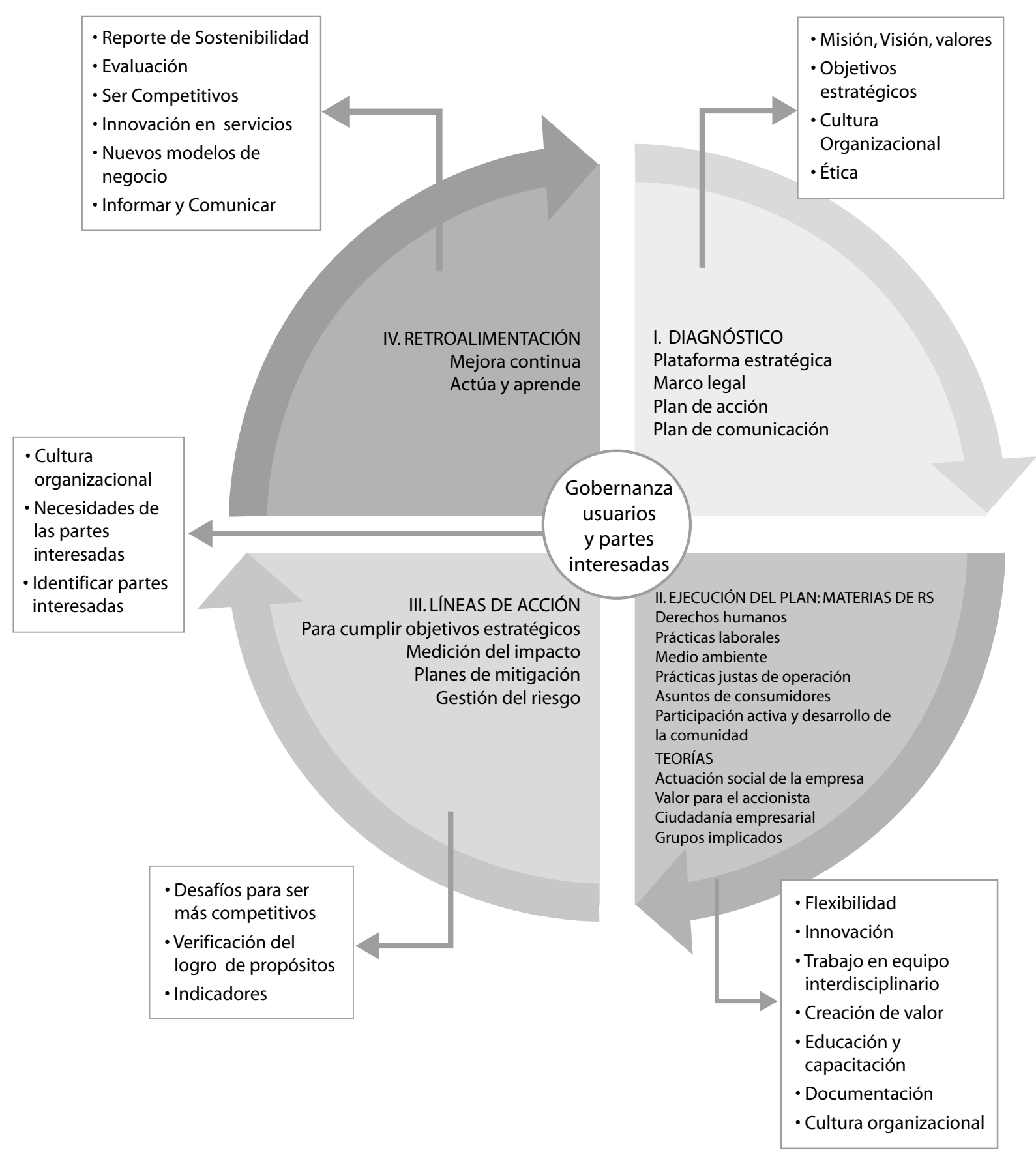

Fuente: elaboración propia 
Con la realización del presente estudio se observa que no solo con la aplicación de la herramienta de gestión de responsabilidad social empresarial se logra una efectiva aplicación de dichas prácticas, sino que debe complementarse con una capacitación continua y divulgación a todo nivel, que involucre una planeación participativa a largo plazo.

El desarrollo de este tipo de proyectos debe seguir en aumento, ya que la universidad debe ser un soporte para las organizaciones externas, destacándose en especial el vínculo universidad-empresa, para brindar apoyo, asesoría, investigación, desarrollo e innovación en herramientas de gestión para las pymes del sector salud.

Para definir el alcance de la responsabilidad social es importante identificar los asuntos fundamentales para la pyme y sus grupos de interés, teniendo en cuenta el marco normativo y el objeto social de las actividades que desarrolla, es por esto que se conoció el perfil de las empresas con las que se trabajó. En la gran encuesta (ANIF, 2011) se observa que el sector de servicios en el que se incluye el sector salud presenta un menor número de áreas de acción frente a los otros sectores de crecimiento de las pymes (servicios y comercio).

Los diferentes servicios que prestan las pymes hacen evidente la importancia de conocer el enfoque de las acciones de responsabilidad social aplicadas para prestar servicios de salud eficientes, eficaces y efectivos que promuevan la promoción y prevención de la salud, bienestar y desarrollo económico en el país.

\section{Enfoques teóricos de las acciones de RSE en las pymes}

La responsabilidad social en las empresas está reflejada en la maximización del valor del accionista, para las pymes del sector salud de la zona suroccidente de
Bogotá, fue desarrollada en un $42 \%$, mostrando una clara tendencia hacia el cumplimiento de lineamientos normativos, es decir que la sustentabilidad de una organización contempla la libertad de empresa, de mercado y la propiedad privada (Melé, 2007), donde la empresa está para crear riqueza y donde el impacto producido por la actividad empresarial es responsabilidad del estado.

Según los resultados, las pymes estudiadas dan prioridad a los accionistas maximizando el factor económico, invirtiendo en iniciativas que garanticen un beneficio en el desarrollo de los objetivos financieros de las organizaciones a largo plazo, dejando en un segundo plano su razón de ser: la salud del usuario y su familia, ya que el enfoque de ciudadanía empresarial corporativa fue del $25 \%$ y el enfoque de actuación social de la empresa del $9 \%$ para las pymes estudiadas del sector salud en esta investigación (figura 5).

De acuerdo a la encuesta de RSE realizada por la ANDI durante el 2012 y 2013, el generar valor para los accionistas fue lo más valorado $(50,5 \%)$ porque un número creciente de empresas considera esta práctica como una meta u objetivo empresarial (Hecking y Tarrazón, 2003; Nejati, Shafaei, Salamzadeh y Daraei, 2011), es decir, es una de las principales motivaciones para implementar una herramienta de gestión de responsabilidad social empresarial para estas empresas. En la herramienta de gestión propuesta (figura 7) se refleja el fortalecimiento de las relaciones con todos los grupos de interés, enmarcando en su plataforma de estrategia y asociado a la actividad de las pymes en todo su valor, no solo dando prioridad al cumplimiento de las normas sino fortaleciendo la gestión social.

La teoría de la ciudadanía empresarial define las acciones sociales responsables y filantrópicas empresariales para contribuir a dar respuesta a determinadas necesidades de la comunidad donde opera la pyme, esto 
se refleja en su actividad económica, el sector salud, siendo esta considerada un derecho fundamental del ser humano. En la herramienta de gestión propuesta se refleja esta teoría en la plataforma estratégica, en el mapa de riesgos, asociados a la actividad de la pyme en toda su cadena de valor y todo lo que se hace más allá del cumplimiento de la legislación y las normas (El Tiempo, 2014).

La teoría de los grupos implicados, según el análisis realizado, hace que la herramienta de gestión (figura 7) se centre en la participación de todas las partes interesadas (comunidades de las zonas de influencia, contratistas, proveedores, consumidores, empleados, accionistas, inversionistas, beneficiarios directos de los planes de inversión), ya que para que las pymes sean competitivas y productivas se debe tener en cuenta estas teorías para generar confianza y también saber cómo nos perciben los demás (clientes, comunidades, sociedad, etc.) (Acosta, Cruz, Salanova y Llorens, 2015).

Lo que menos motiva a las pymes estudiadas es la actuación social de la empresas con un $9 \%$, según la encuesta de la RSE de la ANDI 2012-2013, es el principal obstáculo para el avance de la responsabilidad social de Colombia, ya que existe un desinterés de las causas sociales en un $52 \%$ (ANDI, 2012).

En cuanto a la teoría de orientación de las prácticas RSE que motivan a las pymes del sector salud evaluadas es la actuación social de la empresa la que repercute en una conducta responsable para evitar riesgos y crear reputación corporativa como lo refiere Melé (2007), teniendo en cuenta la tarea económica de la empresa (instalaciones, proveedores, selección del personal, etc.) y los aspectos sociales derivados como la contaminación o el desarrollo profesional de los trabajadores contratados. En la herramienta de gestión propuesta la aplicación de esta teoría se refleja en la plataforma estratégica, en las líneas de acción deseables, en términos de valores y necesidades insatisfechas de la comunidad, (social, ambiental y económico) (figura 8).

Las pymes del sector evaluado aplican un enfoque teórico de las prácticas de responsabilidad social maximizando el valor del accionista y minimizando la acción de la empresa, mostrando la necesidad de aplicar una herramienta de gestión como la propuesta en el presente trabajo, con el fin de orientar sus actividades socialmente responsables para ser sostenibles en el tiempo, competitivas e innovadoras en forma responsable.

La innovación responsable pretende ubicar la creatividad de la organización al servicio de la sostenibilidad, con valores agregados y acumulados de conocimiento, con un modelo de negocio tan innovador que contribuya a superar la inequidad social. Teniendo en cuenta que solo un $23 \%$ de las pymes en Colombia conocen y aplican estrategias de responsabilidad social. La cifra es baja si se tiene en cuenta que alrededor del $85 \%$ de las empresas son clasificadas como pymes en Colombia (ANDI, 2012).

\section{Enfoques prácticos de las acciones de RSE en las pymes}

El enfoque de las prácticas de responsabilidad social orientadas hacia la sociedad influye en el incremento de la competitividad de las pequeñas y medianas empresas (Villegas, 2013; Turyakira, Venter y Smith, 2014). Tales actividades son vistas como el soporte para el enfoque social. Este enfoque es analizado por Inyang (2013) en un estudio donde las estrategias para la práctica de responsabilidad social envuelven al desarrollo de la empresa y comunidad relacionando actividades como líneas de acción, tales como: deportes, salud educación y filantropía. Igualmente, como lo 
argumenta Vargas (2011), la RSE promueve el desarrollo y amplia el rango de garantías ciudadanas que establecen las condiciones de vida de la comunidad y las condiciones organizacionales para materializar el potencial social.

La herramienta de gestión propuesta (figura 7) involucra esta materia fundamental que está en la plataforma estratégica, en las líneas de acción ejecución del plan de acción y en la retroalimentación y mejora continua.

La encuesta de la ANDI del 2012 sobre responsabilidad social muestra que la principal motivación para implementar la herramienta de gestión es mejorar las relaciones con los grupos de interés en 76,7 \% (ANDI, 2012).

El enfoque de responsabilidad social en las pymes evaluadas (figura 6) se presentó en un $28 \%$, tanto para gobernanza como para prácticas laborales. La gobernanza, de acuerdo a la norma ISO 26000:2010, es el sistema por el cual una organización toma e implementa decisiones para lograr sus objetivos y hacer posible un cambio hacia una conducta. Mediante una política de responsabilidad social se crea una cultura, se establecen incentivos para obtener un desempeño positivo, se hace un uso eficiente de recursos, se atienden necesidades de las partes interesadas, se mejora la comunicación de dichas partes y se realiza una revisión continua.

De acuerdo con la ANDI 2012-2013, el $80 \%$ de las empresas cuentan con una política o estrategia de responsabilidad social empresarial; en el 2010 la cifra era del $73 \%$ (ANDI, 2012).

En la herramienta de gestión propuesta (figura 7) aparece ubicada en el centro porque es el factor más importante para articular la responsabilidad social y hacer que las organizaciones se responsabilicen de sus impactos de las actividades que esta realiza. Las prácticas laborales de una organización, según las norma ISO 26000:2010, son todas aquellas prácticas y políticas que involucran a los trabajadores de la propia organización 0 a trabajadores subcontratados. Se incluye la selección y promoción de los trabajadores, traslados, formación de habilidades, salud, seguridad e higiene industrial y cualquier política o práctica que afecte las condiciones de trabajo.

De acuerdo con la encuesta de la ANDI 2012-2013, el principal tema de interés que tienen en cuenta las organizaciones en Colombia para la toma de decisiones en su gestión, es el cliente interno con un $13 \%$. En la herramienta propuesta (figura 7) se encuentra en la plataforma estratégica y es transversal a la organización porque son esenciales para la justicia social, estabilidad y para la paz, son capital humano, ya que los trabajadores no deberían ser tratados como un factor de producción.

En el enfoque práctico de responsabilidad social en las pymes evaluadas (figura 6), se presentó en un $27 \%$ el medio ambiente, de acuerdo con la norma ISO 26000:2010 la organización debe generar impactos en su entorno medioambiental. La utilización de los recursos, la localización física y la producción de residuos y agentes contaminantes son factores que afectan la estabilidad de los ecosistemas.

Según la encuesta de la ANDI 2012-2013, las empresas cuentan con iniciativas por encima de los requisitos de la ley para superar las exigencias del gobierno en materias de: uso de recursos naturales (66\%), manejo de residuos peligrosos $(61,5 \%)$, identificación de impactos ambientales en sus operaciones (59\%), aprovechamiento de residuos sólidos (53,7\%), estándares de vertimientos (46,6\%), estándares de emisiones atmosféricas $(39,2 \%)$, protección de ecosistemas $(32,2 \%)$ y gestión de posconsumo de productos al final de su vida útil (28,6\%) (ANDI, 2012). 
En la herramienta gestión propuesta (figura 7) se encuentra este enfoque en la plataforma estratégica, en las líneas de acción, en la retroalimentación y mejora continua.

El enfoque práctico de responsabilidad social en las pymes evaluadas (figura 6), se presentó en un $26 \%$ en las prácticas justas de operación, de acuerdo a la norma ISO 26000:2010, se refieren a la necesidad de la organización a un comportamiento ético en sus relaciones con otras organizaciones, así como con sus partes interesadas. Para la norma ISO 26000:2010, las prácticas justas de operación pueden ser una útil herramienta para generar resultados socialmente favorables, por ejemplo, proporcionando liderazgo y promoviendo conductas de responsabilidad social en la esfera de influencia de la organización. Según la encuesta de la ANDI 2012-2013, una de las principales motivaciones para implementar una herramienta de gestión es incrementar la reputación organizacional (valor de marca) en un 63,4\% (ANDI, 2012).

Las acciones de este enfoque de $\mathrm{RS}$ se ven reflejadas en la política responsable, donde se promueve la cadena de valor y respeto a los derechos de la propiedad. En la herramienta de gestión propuesta (figura 7) se encuentra en la plataforma estratégica, en el plan de acción y gestión del cambio.

El enfoque práctico de responsabilidad social en las pymes evaluadas (figura 6), se presentó en un $21 \%$ en los asuntos de los consumidores. De acuerdo a la norma ISO 26000:2010 es la importancia de que la organización asuma ciertas responsabilidades con sus consumidores, recomienda tener la responsabilidad de retirar productos del mercado que puedan ocasionar daños, y en caso de que se manejen datos privados, asegurarse de que se mantiene la privacidad de los consumidores.

Según los resultados de la aplicación del cuestionario, predominan prácticas y acciones con el propósito de mejorar la calidad de vida de la comunidad de su entorno, y se fundamenta en el fortalecimiento de las relaciones con los grupos de interés, la articulación con otras organizaciones y el contexto social, es decir, incorpora los intereses de accionistas, de la sociedad y su entorno (Vives, Corral y Isusi, 2005; Martínez, 2010).

En la herramienta de gestión propuesta (figura 7) se encuentra este enfoque en la plataforma estratégica, en el plan de acción y en la gestión del cambio.

El enfoque práctico de responsabilidad social en las pymes evaluadas (figura 6), se presentó en un $19 \%$ con respecto a los derechos humanos, de acuerdo a la norma ISO 26000:2010, son aquellos derechos que posee todo individuo por el simple hecho de haber nacido. Pueden ser civiles, políticos y económicos. Son inherentes a las personas.

Las iniciativas de derechos humanos tienen que ver, por ejemplo, con evitar, mitigar y resolver las situaciones de riesgo que atenten contra tales principios y la no discriminación de grupos vulnerables, entre otros aspectos (ANDI, 2012).

Según la encuesta de la ANDI 2012-2013, de las empresas encuestadas el 41,3\% contratan personas en situación de discapacidad (ANDI, 2012). En la herramienta de gestión propuesta (figura 7) se encuentran líneas de acción orientadas a la inclusión social, a la debida diligencia y a situaciones de riesgos para los derechos humanos.

En general, estas empresas presentan un enfoque social regido por los beneficios económicos, ya que en la parte teórica el valor del accionista muestra un 42 $\%$ de orientación, lo que indica que estas empresas buscan más un beneficio económico. Respecto al enfoque práctico se encontró que solo entre el $19 \%$ y $29 \%$ de las pymes tienen aplicación de las materias fundamentales. 


\section{CONCLUSIONES}

La gestión de la responsabilidad social en las pymes del sector salud debe ser aplicada a todo nivel de la organización, involucrando e impactando positivamente en las partes interesadas, teniendo en cuenta tanto el diagnóstico de los enfoques teóricos y prácticos de la investigación realizada, como la herramienta de gestión social propuesta que involucra las líneas de acción.

El enfoque de la responsabilidad social empresarial de las pymes del sector salud estudiadas fue en la parte teórica el valor del accionista con un $42 \%$, indicando que estas empresas buscan más un beneficio económico y se limitan al cumplimiento de lineamientos normativos, dejando en un segundo plano su razón de ser: la salud del usuario y su familia. Con respecto al enfoque práctico, se encontró que solo entre el $19 \%$ y el $29 \%$ de las pymes tienen aplicación de las materias fundamentales, corroborando que en general estas empresas presentan un enfoque social regido por los beneficios económicos.

La herramienta de gestión social para las pymes vinculadas al sector salud en la zona suroccidente de Bogotá fue realizada teniendo en cuenta el proceso sistemático y cíclico de mejora continua PHVA, que integra el enfoque de responsabilidad social en todos los sistemas de gestión, dándole una sostenibilidad a largo plazo.

La relevancia de este estudio radica en que se hace una determinación de la aplicación de prácticas de responsabilidad social en el sector salud. Un sector que necesita aplicar estas prácticas por varias razones como: la obligación moral, cumplir normas y leyes, el interés en el aporte social que genera y por ser uno de los sectores con mayores exigencias de los órganos de control.

\section{REFERENCIAS}

Acosta, H., Cruz, V., Salanova, M., \& Llorens, S. (2015). Healthy organization: Analysing its meaning based on the Hero model/organizaciones saludables: analizando su significado desde el Modelo Hero. Revista de Psicología Social, 30(2), 323-350.

AsociaciónNacional de Instituciones Financieras-ANIF. (16 de agosto de 2011). Gran encuesta pyme. Bogotá, Colombia. Recuperado de http://www.anif.co/publicaciones/publicaciones-microsectoriales-y-pyme/ gran-encuesta-pyme

Baron, R. A. (2000). Psychological perspectives on entrepreneurship: Cognitive and social factors in entrepreneurs' success. Current directions in psychological science, 9(1), 15-18.

El Tiempo. (abril-junio de 2014). Huella social. Encuesta de responsabilidad social empresarial realizada por la Asociación Nacional de Empresarios - ANDI 2012 2013, (14), 16.

Asociación Nacional de Empresarios Colombianos - ANDI. (2012). Encuesta de Responsabilidad Social Empresarial 2012. Informe. Recuperado de 2013 en: <http://www.andi.com. co/pages/proyectos_paginas/proyectos_detail. aspx?pro_id=69\&Id=6\&clase=8\&Tipo=3 >

Enderle, G. (2003). Competencia global y responsabilidad de las pymes. Madrid: Cortina, Adela.

Giraldo, S. (2011). Formulación de una herramienta para la caracterización de prácticas de responsabilidad social en organizaciones colombianas. (Tesis de maestría). Bogotá: Universidad Santo Tomás.

Hecking, S., \& Tarrazón, M. (2003). The relation between shareholder value orientation and shareholdervalue creation. Universitat Autònoma de Barcelona. 
Instituto Colombiano de Normas Técnicas y Certificación - Icontec. (2010). Guía de responsabilidad social: primera edición noviembre 1 de 2010. Bogotá: Icontec.

Inyang, B. J. (2013). Defining the role engagement of small and medium-sized enterprises (SMEs) in corporate social responsibility (CSR). International business research, 6(5), 123.

Jenkins, H. (2009). A 'business opportunity' model of corporate social responsibility for small and medium-sized enterprises. Business ethics: A european review, 18(1), 21-36.

Ley 905. (2 de agosto de 2004). Por medio de la cual se modifica la Ley 590 de 2000 sobre promoción del desarrollo de la micro, pequeña y mediana empresa colombiana y se dictan otras disposiciones. Diario Oficial $n .{ }^{\circ}$ 45.628. Recuperado de http://www.secretariasenado.gov.co/senado/basedoc/ley_0905_2004.html

Martínez, C. (2010). RSE y lineamientos de investigación desde la perspectiva de la teoría de la gestión. Responsabilidad Social Empresarial. Una reflexión desde la gestión, lo social y ambiental, 1, 1-45.

Melé, D. (2007). Responsabilidad social de la empresa: una revisión crítica a las principales teorías. Ekonomiaz, 65(2), 50-67.

Mora, L. A. E., Miranda, M. C., Espinosa, S. V. A., y Guarín, G. P. (2010). Estado del arte para la línea de investigación en responsabilidad social del Convenio Universidad Santo Tomás e Icontec. Signos, investigación en sistemas de gestión, 2(2), 13.

Nejati, M., Shafaei, A., Salamzadeh, Y., \& Daraei, M. (2011). Corporate social responsibility and universities: A study of top 10 world universities' websites.
Restrepo, R. (2009). Responsabilidad social: nueva teoría, nuevas prácticas. Medellín, Colombia: Editorial Icontec.

Saldaña, A. (2014). Integración regional y sistemas locales de innovación: desafíos para las mipymes. Una perspectiva desde México. Espacio abierto, 23(4).

Trilleros, H. (2015) Caracterización de las iniciativas de responsabilidad social en las organizaciones de Bogotá. (Tesis de maestría). Bogotá: Universidad de Santo Tomás.

Turyakira, P., Venter, E., \& Smith, E. (2014). The impact of corporate social responsibility factors on the competitiveness of small and medium-sized enterprises. South African Journal of Economic and Management Sciences, 17(2), 157-172.

Vargas, G. A. (2011). Corporate social responsibility, citizenship and development. Cuadernos de Administración, 24(43), 177-191.

Villegas, M. G., y Quintanilla, D. A. (2012). Los informes de responsabilidad social empresarial: su evolución y tendencias en el contexto internacional y colombiano. Cuadernos de Contabilidad, 13(32).

Villegas, M. G. (2013). La gestión y la información sobre la responsabilidad social empresarial de las pymes: la necesidad de diferenciación. Contaduría Universidad de Antioquia, (56), 15-40.

Vives, A., Corral, A., y Isusi, I. (2005). Responsabilidad social de la empresa en las pymes de Latinoamérica. Banco Interamericano de Desarrollo. 